\title{
Lattice Green Functions on a Two-Dimensional Rectangular Lattice with Next-Nearest Neighbor Interaction
}

\author{
M. BAK* \\ Faculty of Physics, A. Mickiewicz University, Umultowska 85, 61-614 Poznań, Poland
}

The analytic formula for the on-site Green function on the two-dimensional rectangular lattice is shown for arbitrary energy, both within the band (complex Green function) and outside the band (real Green function), expressed by means of elliptic integrals. The recursion formulae enabling calculation of the Green functions on other lattice sites are shown.

PACS numbers: 02.30.Gp, 02.30.Uu, 02.60.Nm, 71.15.-m, 02.70.Hm, 71.10.Fd

\section{Introduction}

Lattice Green functions appear in many branches of physics, such as: problems of bound states on a lattice, theory of diffusion, random walks, band structure calculations as well as in calculations of resistivity of infinite resistor lattices. They were a subject of examination in sixties, in connection with the investigations of spin waves interactions in the ferromagnetic Heisenberg model (see, e.g. [1]). After a break, an interest in this field slowly grows again, in connection with high-temperature superconductivity theories resorting to Bose-condensation of bound pairs of charges. For two-electron bound pairs in an empty lattice not only we can give the exact eigenenergies and eigenvalues of such pairs but also express them in analytic form - a rare case in the solid state theory. The formulae for lattices with nearest neighbor interactions using elliptic integrals can be found in literature.

An interesting problem is considering next-nearest neighbor interaction, especially in the context of relatively large values of such interactions found in some high-temperature superconductors (on quasi-two-dimensional lattices). These interactions have a large influence on, e.g., phase diagrams of the Hubbard type models or on the evolution of the superconductivity between the limits of BCS and Bose-condensation in these models. Numerically such Green functions on a simple cubic lattice in the direction [111] were calculated by Krompiewski [2], the analytic form for the reciprocal space vector $(\pi, \pi, \pi)$ in the same lattice was given by Bahurmuz [3]; for two-dimensional rectangular lattice the recursion formula was published by Morita [4]. Let us note that the imaginary part of the on-site lattice Green function is proportional to the density of states for a given lattice. In case of the rectangular lattice it

\footnotetext{
* e-mail: mbak@amu.edu.pl
}

was given, e.g., by Xing et al. [5]. Although sometimes the density of states is all we need, in many calculations, for example in problems of bound- and resonant states on a lattice, we also need the real part; often the Green functions connecting different lattice sites, not only the on-site one are useful.

The author does not know any paper describing the full Green function on a two-dimensional rectangular lattice with nearest- and next-nearest hopping. The present paper fills in this gap, showing the on-site Green function for this lattice for arbitrary energy, expressed by means of elliptic integrals. The recursion formulae enabling us to calculate Green functions on other lattice sites are also shown.

\section{Derivation}

We start with the usual definition of the Green function $G\left(\boldsymbol{r}_{i}, \boldsymbol{r}_{j}\right)$ :

$$
(E-H) G=\delta\left(\boldsymbol{r}_{i}-\boldsymbol{r}_{j}\right),
$$

where $E$ is arbitrary energy and $H$ is linear, differential operator on the lattice, e.g., the hopping part of the Hubbard Hamiltonian

$$
H=\frac{1}{N} \sum_{\langle\langle i, j\rangle\rangle} \sum_{\sigma} t_{i, j} c_{i \sigma}^{\dagger} c_{j \sigma},
$$

where $N$ is the number of lattice sites and summation is performed over nearest- and next-nearest neighbors of the rectangular two-dimensional lattice. The Green function can be given by means of the Fourier transform as

$$
G\left(E, \boldsymbol{r}_{i}, \boldsymbol{r}_{j}\right)=\frac{1}{N} \sum_{\boldsymbol{k}} \frac{\mathrm{e}^{\mathrm{i} \boldsymbol{k} \cdot\left(\boldsymbol{r}_{i}-\boldsymbol{r}_{j}\right)}}{E-\varepsilon_{\boldsymbol{k}}},
$$

where

$$
\begin{aligned}
\varepsilon_{\boldsymbol{k}} & =-2 t_{x} \cos k_{x} a_{x}-2 t_{y} \cos k_{y} a_{y} \\
& +4 t_{2} \cos k_{x} a_{x} \cos k_{y} a_{y} .
\end{aligned}
$$

In general, in the continuous limit (lattice constants $a_{x}$ 
and $a_{y}$ omitted after a trivial variable change):

$$
\begin{aligned}
& G_{i, j}(E)=\frac{1}{(2 \pi)^{2}} \iint_{-\pi}^{\pi} \mathrm{d} k_{x} \mathrm{~d} k_{y} \\
& \quad \times \frac{\mathrm{e}^{\mathrm{i} \boldsymbol{k} \cdot \boldsymbol{r}_{i}} \mathrm{e}^{-\mathrm{i} \boldsymbol{k} \cdot \boldsymbol{r}_{j}}}{E-\gamma_{1} \cos k_{x}-\gamma_{2} \cos k_{y}-\gamma_{3} \cos k_{x} \cos k_{y}} .
\end{aligned}
$$

When we deal with the infinite lattice, the Green function is in fact site-independent and we can replace it by the one depending on the relative indexing

$$
\begin{aligned}
& G_{m, n}(E)=\frac{1}{(2 \pi)^{2}} \iint_{-\pi}^{\pi} \mathrm{d} k_{x} \mathrm{~d} k_{y} \\
& \quad \times \frac{\mathrm{e}^{\mathrm{i} m k_{x}} \mathrm{e}^{\mathrm{i} n k_{y}}}{E-\gamma_{1} \cos k_{x}-\gamma_{2} \cos k_{y}-\gamma_{3} \cos k_{x} \cos k_{y}},
\end{aligned}
$$

where $\boldsymbol{r}_{i}-\boldsymbol{r}_{j}=\left(m a_{x}, n a_{y}\right)$ (before variable change). $\gamma_{3}=0$ corresponds to nearest-neighbor hopping. In case of the problem of bound states the coefficients $\gamma_{n}$ can take more complicated forms and depend not only on the hopping integrals $t_{i, j}$ but also on the momentum $\boldsymbol{P}$ of the center of mass of the bound pair. In that case the kinetic energy would be more complicated than Eq. (4); Eq. (5) would correspond to the Green function on $\Gamma-X$ line then, i.e., to $P_{y}=0$. After partial integration the simplest of the integrals of the type given by Eq. (6), $G_{0,0}$, takes the form

$$
G_{0,0}=\frac{2}{\pi} \int_{0}^{\infty} \frac{\operatorname{sgn}[f(t)]}{\sqrt{a t^{4}+b t^{2}+c}} \mathrm{~d} t
$$

where

$$
\begin{aligned}
& a=\left(E+\gamma_{2}\right)^{2}-\left(\gamma_{1}-\gamma_{3}\right)^{2}, \\
& b=2\left(E^{2}-\gamma_{1}^{2}-\gamma_{2}^{2}+\gamma_{3}^{2}\right), \\
& c=\left(E-\gamma_{2}\right)^{2}-\left(\gamma_{1}+\gamma_{3}\right)^{2}, \\
& f=E+\gamma_{1}+\left(\gamma_{3}-\gamma_{2}\right)\left(1-t^{2}\right) /\left(1+t^{2}\right) .
\end{aligned}
$$

This form is ready for the transformation into the elliptic integral. The result will depend on the solutions $t_{1}^{2}$ and $t_{2}^{2}$ of the biquadratic polynomial in the denominator of Eq. (7):

$$
\begin{aligned}
& t_{1}^{2}=\frac{-E-\gamma_{1}+\gamma_{2}-\gamma_{3}}{E+\gamma_{1}+\gamma_{2}-\gamma_{3}}, \\
& t_{2}^{2}=\frac{-E+\gamma_{1}+\gamma_{2}+\gamma_{3}}{E-\gamma_{1}+\gamma_{2}+\gamma_{3}},
\end{aligned}
$$

on whether they are positive or negative. The signum in the numerator can be safely omitted, except from the case of both positive roots, when it can cause disappearing of the real part of the Green function, as will be shown later.

At the energies $E$ where $t_{1}^{2}$ or $t_{2}^{2}$ change sign the analytical form of $G_{0,0}$ also changes. For energies beyond the band, where both solutions $t_{1}^{2}$ and $t_{2}^{2}$ are negative (and thus Green function Eq. (7) has no singularities), $G_{0,0}$ is given by

$$
G_{0,0}^{--}= \pm \frac{2}{\pi \sqrt{a}} \frac{1}{\sqrt{\left|t_{\max }^{2}\right|}} \mathcal{K}\left(1-\frac{t_{\min }^{2}}{t_{\max }^{2}}\right),
$$

which is purely real. The minus sign is for the negative energies, plus - for the positive ones; $t_{\min }^{2}$ and $t_{\max }^{2}$ denote this one of the solutions $t_{1}^{2}$ and $t_{2}^{2}$, of the smaller and of the larger absolute value respectively and $\mathcal{K}(\ldots)$ is complete integral of the first kind (a convention of denoting $K\left(k^{2}\right)$ by a squared modulus is used).

The band extends between the minimal and the maximal value in the set $\mathcal{S}$ of four elements: $\mathcal{S}=\left\{\gamma_{1}+\gamma_{2}+\right.$ $\left.\gamma_{3},-\gamma_{1}-\gamma_{2}+\gamma_{3},-\gamma_{1}+\gamma_{2}-\gamma_{3}, \gamma_{1}-\gamma_{2}-\gamma_{3}\right\}$. At these energies the Green function has singularities or jumps and $t_{1}^{2}$ and $t_{2}^{2}$ become zero or diverge. The imaginary part of Green function may also diverge at the energy equal to $-\gamma_{1} \gamma_{2} / \gamma_{3}$, if both roots (12) and (13) are positive and the discriminant of the polynomial under the square root in the denominator of equation (7) is positive between $t_{1}$ and $t_{2}$. In that case, the imaginary part of $G_{0,0}$ has four jumps and one singularity; the real part of Green function has a jump at the energy of the singularity, it is negative for energies smaller than the energy of the jump and positive for larger energies. If the discriminant is negative between $t_{1}>0$ and $t_{2}>0$ then the imaginary part of LGF has two jumps and two singularities. The real part of $G_{0,0}$ between the singularities is then zero, is negative for smaller energies and positive for larger ones.

For some of the gammas being of the same absolute value the Green function simplifies. If all three $\gamma_{\mathrm{n}}$ 's (for $n=1,2,3)$ are of the same absolute value there are only two different values contained in the set $\mathcal{S}$ - they mark the band boundaries. The imaginary part of $G_{0,0}$ has one jump and one singularity then. If the two of $\gamma_{n}$ 's are of the same absolute value, then there are three different elements in $\mathcal{S}$, dividing the band into two regions $(\operatorname{Im}(G)$ has two jumps and one singularity); when all $\gamma_{\mathrm{n}}$ 's are different the band is divided into three regions by four different values from $\mathcal{S}$ (or four, when the singularity in $-\gamma_{1} \gamma_{2} / \gamma_{3}$ appears).

Within the band at least one of $t_{1}^{2}$ and $t_{2}^{2}$ or both are positive. The analytic solutions in these cases are given below

$$
\begin{aligned}
& G_{0,0}^{+-}=\frac{ \pm 2}{\pi \sqrt{a}} \frac{1}{\sqrt{t_{\mathrm{p}}^{2}+\left|t_{\mathrm{n}}^{2}\right|}} \\
& \quad \times\left[\mathcal{K}\left(\frac{\left|t_{\mathrm{n}}^{2}\right|}{\left|t_{\mathrm{n}}^{2}\right|+t_{\mathrm{p}}^{2}}\right)-\mathrm{i} \mathcal{K}\left(\frac{t_{\mathrm{p}}^{2}}{\left|t_{\mathrm{n}}^{2}\right|+t_{\mathrm{p}}^{2}}\right)\right], \\
& G_{0,0}^{++}=\frac{ \pm 2}{\pi \sqrt{a}} \frac{1}{\sqrt{t_{\max }^{2}}}\left(g_{r}^{++}+i g_{i}^{++}\right),
\end{aligned}
$$

where $g_{r}^{++}=0$ or $2 \mathcal{K}\left(\frac{t_{\text {min }}^{2}}{t_{\text {max }}^{2}}\right), g_{i}^{++}=-\mathcal{K}\left(1-\frac{t_{\text {min }}^{2}}{t_{\text {max }}^{2}}\right)$, and $t_{p}^{2}$ and $t_{n}^{2}$ are the positive and the negative solutions respectively. Whether $g_{r}^{++}$is zero or nonzero depends on the sign of discriminant of polynomial in Eq. (7), as was described before. The solutions Eqs (15) and (16) are accurate only up to the sign. As far as the actual signs of $G_{0,0}$ within the band are concerned they are as follows: $\operatorname{Im}\left(G_{0,0}\right)$ is taken always positive, what is equivalent to calculating the resolvent in Eq. (1) with the negative, infinitesimal, imaginary part added to the energy: $E-i \varepsilon$, $0<\varepsilon \ll 1$. The rules of deciding the sign of $\operatorname{Re}\left(G_{0,0}\right)$ 
were described before. In case all three gammas are of the same absolute value the sign of $\operatorname{Re}\left(G_{0,0}\right)$ is the same as the sign of the product $\gamma_{1} \times \gamma_{2} \times \gamma_{3}$.

The conditions for $t_{1}^{2}$ and $t_{2}^{2}$ can be expressed in the form of conditions for the energy $E$ but they become extremely cumbersome then.

\section{Recurrence relations}

Once we have $G_{0,0}$ we need other Green functions, between other lattice sites. We could calculate them like Eq. (7) but with the appropriate exponenses in the numerator. Another way is to try to find the recurrence relations and check, whether there is a finite number of Green functions enabling us to calculate all the others.

The defining recurrence is as follows:

$$
\begin{aligned}
& 2 E G_{m, n}=-2 \delta_{m, 0} \delta_{n, 0}+\gamma_{2}\left(G_{m, n-1}+G_{m, n+1}\right) \\
& \quad+\gamma_{1}\left(G_{m-1, n}+G_{m+1, n}\right) \\
& \quad+\frac{\gamma_{3}}{2}\left(G_{m+1, n+1}+G_{m-1, n-1}\right. \\
& \left.\quad+G_{m-1, n+1}+G_{m+1, n-1}\right) .
\end{aligned}
$$

Another one can be obtained by differentiating by $E$ the previous relation

$$
\begin{aligned}
& 2 G_{m, n}+2 E G_{m, n}^{\prime}=\gamma_{2}\left(G_{m, n-1}^{\prime}+G_{m, n+1}^{\prime}\right) \\
& \quad+\gamma_{1}\left(G_{m-1, n}^{\prime}+G_{m+1, n}^{\prime}\right) \\
& \quad+\frac{\gamma_{3}}{2}\left(G_{m+1, n+1}^{\prime}+G_{m-1, n-1}^{\prime}\right. \\
& \left.\quad+G_{m-1, n+1}^{\prime}+G_{m+1, n-1}^{\prime}\right) .
\end{aligned}
$$

$G_{m, n}^{\prime}$ is given by the equation analogous to Eqs. (5) or (6), just with the denominator squared and minus in front.

We can obtain two more relations by partial integration of the Green function Eq. (6) by parts [6]. Performing the first integral with respect to $x$ or $y$ yields relations including $m$ and $n$, respectively

$$
\begin{aligned}
0 & =2 m G_{m, n}-\gamma_{1}\left(G_{m+1, n}^{\prime}-G_{m-1, n}^{\prime}\right) \\
& -\frac{\gamma_{3}}{2}\left(G_{m+1, n+1}^{\prime}-G_{m-1, n-1}^{\prime}-G_{m-1, n+1}^{\prime}\right. \\
& \left.+G_{m+1, n-1}^{\prime}\right), \\
0 & =2 n G_{m, n}-\gamma_{2}\left(G_{m, n+1}^{\prime}-G_{m, n-1}^{\prime}\right) \\
& -\frac{\gamma_{3}}{2}\left(G_{m+1, n+1}^{\prime}-G_{m-1, n-1}^{\prime}+G_{m-1, n+1}^{\prime}\right. \\
& \left.-G_{m+1, n-1}^{\prime}\right) .
\end{aligned}
$$

using the symmetry property

$$
G_{m, n}=G_{-m, n}=G_{m,-n}=G_{-m,-n},
$$

obtaining equations for non-negative indices only.

\subsection{Constructing closed sets of equations}

As can be seen by inspection, we cannot construct a closed set of equations of the type of Eq. (17) for any fixed $m$ or $n$. Yet assembling the derivative equations of the type (18)-(20) for the Green functions $G_{m, n}$ within $z$-th "layer", i.e., for $m=z$ with $n \leq z$ and $n=z$ with $m \leq z$, yields a closed set of equations for $G_{m, n}$ 's from $z$-th layer and derivatives $G_{m, n}^{\prime}$ 's from layers $z-1, z$ and $z+1$. Starting from second layer these equation sets are overcomplete and we can calculate all the variables (the Green functions and its derivatives) of a given layer, knowing only the variables from the lower layers. Thus we can finally obtain all the Green functions and its derivatives based only on the knowledge of the four parameters from the first and zeroth layer, e.g.: $G_{0,0}$, $G_{1,0}, G_{0,1}$ and $G_{0,0}^{\prime}$.

\section{Conclusions}

In conclusion, a closed analytic form of the on-site Green function $G_{0,0}(E)$ on a two-dimensional rectangular lattice was shown for arbitrary energy. The recurrence formulae for $G_{m, n}$ 's as well as for their derivatives were given and a way to calculate Green function on arbitrary site based on the knowledge of only four parameters was shown.

\section{References}

[1] R.G. Boyd, J. Callaway, Phys. Rev. 140, A1621 (1965).

[2] S. Krompiewski, Acta Phys. Pol. A 53, 845 (1978).

[3] A.A. Bahurmuz, Acta Phys. Pol. A 57, 565 (1980).

[4] T. Morita, J. Phys. A, Math. Gen. 8, 478 (1975).

[5] D.Y. Xing, M. Liu, C.D. Gong, Phys. Rev. B 44 12525 (1991).

[6] T. Horiguchi, T. Morita, J. Phys. C, Solid State Phys. 8, L232 (1975).

We can simplify equations for fixed values of $m$ and $n$ 